\title{
Improved local lattice Monte Carlo simulation for charged systems
}

Jian Jiang, and Zhen-Gang Wang

Citation: The Journal of Chemical Physics 148, 114105 (2018); doi: 10.1063/1.5023491

View online: https://doi.org/10.1063/1.5023491

View Table of Contents: http://aip.scitation.org/toc/jcp/148/11

Published by the American Institute of Physics

\section{Articles you may be interested in}

Constructing first-principles phase diagrams of amorphous $\mathrm{Li}_{x} \mathrm{Si}$ using machine-learning-assisted sampling with an evolutionary algorithm

The Journal of Chemical Physics 148, 241711 (2018); 10.1063/1.5017661

Molecular-dynamics study of propane-hydrate dissociation: Fluctuation-dissipation and non-equilibrium analysis

The Journal of Chemical Physics 148, 114504 (2018); 10.1063/1.5018192

Quantum theory of multiscale coarse-graining

The Journal of Chemical Physics 148, 102335 (2018); 10.1063/1.5010270

Non-covalent interactions across organic and biological subsets of chemical space: Physics-based potentials parametrized from machine learning

The Journal of Chemical Physics 148, 241706 (2018); 10.1063/1.5009502

Comprehensive representation of the Lennard-Jones equation of state based on molecular dynamics simulation data

The Journal of Chemical Physics 148, 114505 (2018); 10.1063/1.5021560

An atomistic fingerprint algorithm for learning ab initio molecular force fields

The Journal of Chemical Physics 148, 034101 (2018); 10.1063/1.5008630

\section{AIP | the Joumal of Chemical Physics}




\title{
Improved local lattice Monte Carlo simulation for charged systems
}

\author{
Jian Jiang and Zhen-Gang Wang ${ }^{\text {a) }}$ \\ Division of Chemistry and Chemical Engineering, California Institute of Technology, Pasadena, \\ California 91125, USA
}

(Received 24 January 2018; accepted 27 February 2018; published online 16 March 2018)

\begin{abstract}
Maggs and Rossetto [Phys. Rev. Lett. 88, 196402 (2002)] proposed a local lattice Monte Carlo algorithm for simulating charged systems based on Gauss's law, which scales with the particle number $N$ as $O(N)$. This method includes two degrees of freedom: the configuration of the mobile charged particles and the electric field. In this work, we consider two important issues in the implementation of the method, the acceptance rate of configurational change (particle move) and the ergodicity in the phase space sampled by the electric field. We propose a simple method to improve the acceptance rate of particle moves based on the superposition principle for electric field. Furthermore, we introduce an additional updating step for the field, named "open-circuit update," to ensure that the system is fully ergodic under periodic boundary conditions. We apply this improved local Monte Carlo simulation to an electrolyte solution confined between two low dielectric plates. The results show excellent agreement with previous theoretical work. Published by AIP Publishing. https://doi.org/10.1063/1.5023491
\end{abstract}

\section{INTRODUCTION}

Electrostatic interactions are fundamental to physics, chemistry, material science, atmospheric science, and biology. Due to the long range nature, simulating systems with electrostatic interactions has been computationally demanding. ${ }^{1}$ Furthermore, the use of periodic boundary conditions (PBCs) in typical simulation of finite-sized systems introduces image interactions between the periodic images, which must be treated with care. The most commonly used method is the Ewald summation algorithm, which has a computational complexity of $O\left(N^{3 / 2}\right)$, where $N$ is the number of charged particles. $^{2,3}$ A large amount of effort has been put into optimizing the calculation of electrostatic energy; ${ }^{4-15}$ however, simulation of a charged system with large sizes remains CPU (central processing unit) intensive task. While parallelization techniques can in principle accelerate the simulation and have shown promises in molecular dynamics simulations, ${ }^{16}$ these techniques are less efficient for Monte Carlo simulations, due to the need to calculate the non-local interaction energy after every single trial move. A lattice Monte Carlo algorithm, based on the use of the electric field $\mathbf{E}(\mathbf{r})$ for Coulomb systems, was introduced by Maggs and collaborators. ${ }^{17-19}$ The field $\mathbf{E}$ in this algorithm is purely "local" on an interpolating grid. Although a benchmark study of the molecular dynamics version of this method based on a single core revealed no speed advantages over traditional fast electrostatic (Fourier-based) algorithms, excellent efficiencies are obtained on parallel clusters. ${ }^{9,20}$ Therefore, this local method can be expected to offer a promising strategy for coding efficient parallel Monte Carlo simulation programs.

The local Monte Carlo simulation algorithm proposed by Maggs and co-workers ${ }^{17-19}$ applies Metropolis sampling

a)Electronic mail: zgw@caltech.edu together with Gauss's law constraint for a fluctuating electric field. The configuration sampling involves two kinds of updates: (1) the electric field on the grid for a given particle configuration, subject to the constraint of Gauss's law, and (2) the position of the charged particles, also subject to the constraint of Gauss's law. The field updates generate the physical electric field as the average of the fluctuating field by the Metropolis sampling for a given particle configuration while particle position updates generate a new configuration of the mobile ions.

The original algorithm of Maggs and Rossetto suffers from low acceptance rates associated with moving the charged particles. ${ }^{17,19}$ The low acceptance rate results from the large disruption in the value of the local field near the ion that is being moved, which in turn leads to a large energy penalty. To overcome this shortcoming, Duncan et al. proposed a coupled update procedure ${ }^{21}$ where each update of the particle position is accompanied by a simultaneous update of the fields in the vicinity of the ion. Although this method resulted in a significant improvement in the acceptance rate for monovalent ions at relatively low concentration and/or high temperature, extension of this method to systems with stronger interactions (such as multivalent ions, high concentration/low temperature) is difficult. An alternative charge spreading method was proposed by Levrel and Maggs. ${ }^{19}$ In this method, the charge on the particle is first spread evenly to the neighboring $w^{3}$ sites. The particle move now consists of collectively shifting the charge distribution by a lattice unit, followed by a redistribution of the charge back to the central single site. In this work, we propose a simpler algorithm to improve the acceptance rate for particle moves based on the superposability of electric field.

Another issue concerns the ergodicity in the field sampling. ${ }^{17,18}$ For systems with non-periodic boundary conditions, it was demonstrated that the so-called "plaquette" updating is 
sufficient to ensure ergodicity in the sampling. However, in the case of PBCs, additional global moves are required in order to achieve full ergodicity, as first noted by Maggs and Rossetto. ${ }^{17}$ Reference 17 proposed adding a uniform fluctuating field to supplement the "plaquette" updating. However, as we will show in Sec. III B, such a uniform fluctuating field is insufficient to ensure ergodicity; we propose a new scheme that does result in ergodic sampling of the field.

We begin in Sec. II with a brief recapitulation of the salient points in the theoretical formulation of the Maggs-Rossetto local Monte Carlo algorithm. In Sec. III, we discuss the implementation of this local algorithm. We propose an efficient and flexible method to improve the acceptance rate for the particle moves in Sec. III A and introduce an "open-circuit" updating method to ensure ergodicity in the field updates. In Sec. IV, we apply our improved algorithm to study the image charge effects on the electric double layer. Section V provides a brief summary and some concluding remarks.

\section{THEORETICAL FORMULATION}

We start with the expression for the electrostatic energy in a uniform dielectric background in terms of the electric field $\mathbf{E}$,

$$
\mathcal{U}=\epsilon \int \mathrm{d} \mathbf{r} \frac{\mathbf{E}^{2}}{2}
$$

where $\epsilon=\epsilon_{0} \epsilon_{\mathrm{r}}$ is the permittivity. The electric field $\mathbf{E}$ is constrained by Gauss's law

$$
\nabla \cdot \mathbf{E}-\rho / \epsilon=0
$$

and the static condition

$$
\nabla \times \mathbf{E}=0
$$

In the Maggs-Rossetto algorithm, the static condition is lifted and replaced by integration over a fluctuating field. To demonstrate this, we consider the following "partition function" for a fixed configuration $\left(\left\{\mathbf{r}_{i}\right\}\right)$ of the mobile charges $q_{i}$ at inverse temperature $\beta$,

$$
\mathcal{Z}\left(\left\{\mathbf{r}_{i}\right\}\right)=\int \mathrm{D} \mathbf{E} \exp \left[-\frac{\beta \epsilon}{2} \int \mathrm{d} \mathbf{r} \mathbf{E}^{2}\right] \prod_{\mathbf{r}} \delta\left[\nabla \cdot \mathbf{E}-\frac{\rho}{\epsilon}\right],
$$

where the charge density $\rho(\mathbf{r})$ is shorthand for

$$
\rho(\mathbf{r})=\sum_{i} q_{i} \delta\left(\mathbf{r}-\mathbf{r}_{i}\right) .
$$

Note that because the field $\mathbf{E}$ in Eq. (2.4) does not satisfy the rotation-free condition Eq. (2.3), it is not the physical electric field but a fluctuating field. The physical electric field is obtained by averaging the fluctuating field for a given particle configuration. However, to economize notation, we will still use $\mathbf{E}$ to denote the fluctuating field hereafter. Because both the energy [Eq. (2.1)] and Gauss's law constraint are local, the formulation of the "partition function" Eq. (2.4) is manifestly local.

The field that satisfies Gauss's law Eq. (2.2) can be written generally as

$$
\mathbf{E}=-\nabla \phi+\nabla \times \mathbf{Q}
$$

where $\phi$, the solution of Poisson equation $\nabla^{2} \phi=-\rho / \epsilon$, is unique to within a constant for a specified dielectric constant and boundary condition and $\mathbf{Q}$ is an arbitrary vector. Note that while the first term in Eq. (2.6) is rotation-free $(\nabla \times \nabla \phi=0)$, the second term is only divergence-free or source-free $(\nabla \cdot \nabla \times \mathbf{Q}=0)$. Because of the decomposition of $\mathbf{E}$, Eq. (2.6), the "partition function" Eq. (2.4) can now be written as

$$
\begin{aligned}
\mathcal{Z}\left(\left\{\mathbf{r}_{i}\right\}\right)= & \exp \left[-(\beta \epsilon / 2) \int \mathrm{d} \mathbf{r}(\nabla \phi)^{2}\right] \\
& \times \int \mathcal{D} \mathbf{E}_{t} \exp \left[-(\beta \epsilon / 2) \int \mathrm{d} \mathbf{r} \mathbf{E}_{t}^{2}\right],
\end{aligned}
$$

where $\mathbf{E}_{t}=\nabla \times \mathbf{Q}$ is the transverse part of the field $\mathbf{E}$ and $\mathcal{D} \mathbf{E}_{t}=\prod_{\mathbf{r}} \delta\left(\nabla \cdot \mathbf{E}_{t}\right) \mathrm{DE} \mathbf{E}_{t}$. For a configuration-independent dielectric function $\epsilon$, the last integral is a constant and we obtain the key result that the "partition function" over the fluctuating field $\mathbf{E}$ yields just the Boltzmann weight for the electrostatic interaction up to a constant factor.

We reiterate that although $\mathbf{E}$ is a fluctuating field controlled by the temperature, its average at any fixed particle configuration (i.e., fixed charge distribution) is the physical electric field satisfying both Gauss's law and the static condition. In particular, at zero temperature, the electric field generated by this local algorithm is the physical electric field. As long as Gauss's law is strictly satisfied, an integration over $\mathbf{E}_{t}$ will generate the correct Coulomb interactions between the particles. Note that the "partition function" $\mathcal{Z}\left(\left\{\mathbf{r}_{i}\right\}\right)$ in Eq. (2.4) samples only the field degrees of freedom. The full partition function is obtained by further integration over the particle positions,

$$
\mathcal{Q}=\frac{1}{N ! v^{N}} \int \prod_{i} \mathrm{~d} \mathbf{r}_{i} \mathcal{Z}\left(\left\{\mathbf{r}_{i}\right\}\right)
$$

where $v$ is some volume scale, which can be taken as the cube of the thermal de Broglie wavelength. The exact specification of $v$ is inconsequential as it merely shifts the free energy by a constant.

\section{IMPLEMENTATION AND IMPROVEMENT}

The partition function Eq. (2.8) involves two kinds of degrees of freedom: the field $\mathbf{E}$ and the particle position $\left\{\mathbf{r}_{i}\right\}$. For a fixed $\left\{\mathbf{r}_{i}\right\}$, sampling of $\mathbf{E}$ is achieved by a Metropolis algorithm subject to Gauss's law. However, a particle move involving the charge redistribution must also be subjected to Gauss's law. Maggs and Rossetto devised the following Monte Carlo schemes to affect these two moves. Here we briefly recapitulate the lattice version of their algorithm, although Rottler and Maggs $^{22}$ have designed an off-lattice version (based on interpolation method).

In the lattice mode, the system is discretized by placing particles with charge $q_{i}$ on $N=(L / a)^{3}$ sites, indexed as $\{i\}$, of a cubic, periodic simulation cell of volume $L^{3}$, where $a$ is the mesh size. The Cartesian components of the electric field $\mathbf{E}=\left\{E_{i, j}\right\}$ are associated with the $3 N$ links of the lattice, where $E_{i, j}$ is used to denote a local contribution to electric flux leaving lattice site $i$ towards $j$, and $E_{i, j}=-E_{j, i}$. It is convenient to consider Gauss's law in the equivalent integral form on 
lattice

$$
a^{2} \sum_{j} E_{i, j}=q_{i} / \epsilon
$$

where the summation is over the total electric flux leaving the site $i$ towards the six neighbor sites. The corresponding Poisson equation for the electrostatic potential $\varphi_{i}$ is

$$
a \sum_{j}\left(\varphi_{i}-\varphi_{j}\right)=q_{i} / \epsilon
$$

The solution of Eq. (3.2) subject to the appropriate boundary condition constitutes the exact solution to the lattice charged systems, which serves as a benchmark for comparing the accuracy of the different algorithms.

We first discuss the particle update. The key consideration is to displace a charged particle from one site to another without violating Gauss's law. The original scheme by Maggs and Rossetto ${ }^{17}$ starts with an electric field configuration that satisfies Gauss's law. Then one randomly chooses a charged particle, say on site $i$, and shifts it to a neighboring $j$; see Fig. 1(a). The Gauss law constraint is satisfied at both sites for the new configuration if the field variable associated with the link between sites $i$ and $j$ is updated following the rule $E_{i, j} \rightarrow E_{i, j}-q_{i} /\left(a^{2} \epsilon\right)$. Despite the appealing simplicity of this recipe, in physically relevant systems with a large Bjerrum length $\left(l_{B} \approx 7 \AA\right.$ or larger), this method proved to be impractical due to the extremely low acceptance rate. This low acceptance rate results from the large energy penalty associated with the large disruption in the field configuration because each shift in the position of the charged particle generates an energy cost of the order of $\Delta U=q_{i}^{2} /(2 a \epsilon)$. For a typical system (i.e., aqueous solution at room temperature) with univalent charge $\left(z_{i}= \pm 1\right)$, the acceptance rates, $e^{-\beta \Delta U}$, is estimated to be on the order $10^{-4}$.

To mitigate the large energy change on the affected link associated with the particle move, Duncan et al. proposed a coupled updating procedure that involves updating the 12 neighboring links in addition to the one in question. ${ }^{21}$ The scheme is illustrated in Fig. 1(b) for moving a charged particle from site 1 to site 2 . The field on the central link, $E_{1,2}$, is modified by $E_{1,2}+(4 \eta-1) q_{1} /\left(a^{2} \epsilon\right)$, and the field on the 12 neighboring links is changed by $\eta q_{1} /\left(a^{2} \epsilon\right)$, where $\eta$ can be chosen randomly or be assigned a fixed value. This coupled procedure increases the acceptance rate for moving a monovalent particle (for the Bjerrum length corresponding to aqueous

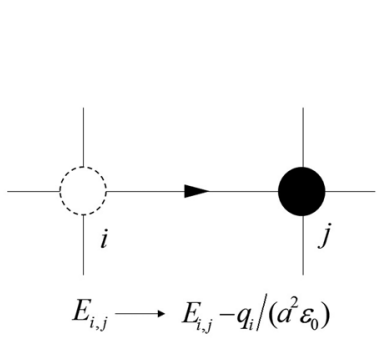

(a)

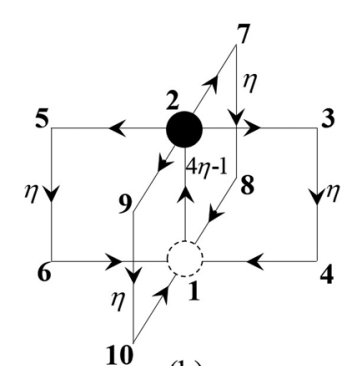

(b)
FIG. 1. (a) Displacement of the charged particle from site $i$ to site $j$, along with the update of the electric variable $E_{i, j}$ to $E_{i, j}-q_{i} /\left(a^{2} \epsilon\right)$. (b) The local field environment for coupled update particle move. $\eta$ is the increment in the field on the links. solution at room temperature) to 0.13 from $10^{-4}$. It is possible to extend the method to include the next-nearest neighbor links; the total number of links is now 63 . We have tested this 63-link coupled scheme and found that the acceptance rate can reach 0.5. However, bookkeeping is quite cumbersome for this 63-link scheme, and the acceptance rate for a larger Bjerrum length or higher-valent ions remains unsatisfactorily low. An alternative method was proposed by Levrel and Maggs. ${ }^{19}$ These authors introduced a 3-step procedure as follows. The charge is first spread evenly onto $w^{3}$ sites, resulting in a subcharge of $q_{i} / w^{3}$ on each of the affected sites. Then the entire charge distribution is shifted by one lattice unit, with simultaneous updating of the field on all the affected links. Finally, the charge is re-assembled onto the shifted central site. The energy cost of this charge spread method for the overall motion of the charged particle is estimated to be $q_{i}^{2} /\left(2 w^{3} a \epsilon\right)$. We refer the interested readers to Ref. 19 for detailed description of this method.

We now discuss the sampling of the fluctuating field $\mathbf{E}$. Since the particle moves are constrained by Gauss's law, which has already included the effect of charge-source, it is only necessary to sample the source-free part of the fluctuating field, $\mathbf{E}_{t}$ (the transverse field). Similar to the Wilson loop in lattice QCD (quantum chromodynamics), ${ }^{23}$ Maggs and Rossetto proposed a simple scheme for sampling the transverse field $\mathbf{E}_{t}$ by modifying one of the $3 N$ plaquettes (each consisting of four links), chosen at random. An update of the field on the four links is achieved by adding an increment $\Delta$ by following the sequence of arrows [see Fig. 2(a)], where $\Delta$ is uniformly distributed between $-\Delta_{0}$ and $\Delta_{0}$. This update modifies the electric field by a pure "closed circuit" such that Gauss's law is automatically satisfied. This four link plaquette algorithm can be extended to $n$ links, where $n$ varies stochastically during the updating. This $n$-link method can be realized by using the cluster Monte Carlo algorithm (the so-called worm update) which was proposed by Alet and Sørensen for the quantum rotor model. ${ }^{24}$ These worm updates make use of a biased random walk to generate a "closed circuit" which includes $n$ links [see Fig. 2(b)]. The field on the $n$ links is updated by adding a random value $\Delta$, where $\Delta \in\left[-\Delta_{0}, \Delta_{0}\right]$ and $\Delta_{0}$ is chosen to make the total number of steps (links) $n$ in a closed-loop walk to be on the order of the

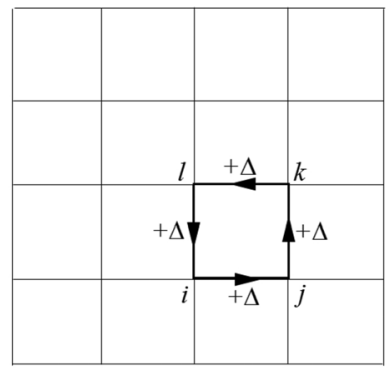

(a)

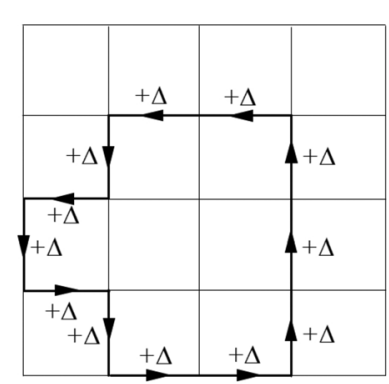

(b)
FIG. 2. Two-dimensional illustration of the field updating schemes. (a) Plaquette scheme: The four field links associated with a single plaquette form a closed circuit. The four links are updated according to the rule: $E_{i, j} \rightarrow E_{i, j}$ $+\Delta, E_{j, k} \rightarrow E_{j, k}+\Delta, E_{l, i} \rightarrow E_{l, i}+\Delta$, where the increment $\Delta$ is chosen randomly within $\left[-\Delta_{0}, \Delta_{0}\right]$; (b) closed-circuit worm update with $n$ links. The rule for the field update is similar to that for the plaquette. 
linear dimension of the lattice. This worm updating method has been shown to be quite efficient for equilibrating the electric field. Thermodynamically, the worm updating method and the four-link plaquette method are equivalent since a worm update can be obtained by multiple plaquette updates.

Having described the current status in the implementation of the Maggs-Rossetto algorithm, we now propose two improvements, focused, respectively, on the particle move and field update.

\section{A. Particle move}

The parameter $w$ in the charge spread method of Levrel and Maggs ${ }^{19}$ is an adjustable factor for regulating the acceptance rate; higher acceptance rate can be obtained by increasing $w$. The key idea in this as well as in the coupled-update method of Duncan $e t \mathrm{al}^{21}$ is to delocalize the energy penalty associated with the central link for the particle move to neighboring links. Following this idea, here we propose a simpler algorithm by making use of the superposition principle of the electric field. We illustrate our method in two-dimension. We consider a particle with charge $q_{i}$ that is shifted from site $i$ to site $j$ (see Fig. 3). We denote the electric field configuration as $\mathbf{E}_{\text {old }}$ before the particle move and $\mathbf{E}_{\text {new }}$ after the move. The difference is $\widehat{\mathbf{E}}=\mathbf{E}_{\text {new }}-\mathbf{E}_{\text {old }}$, which we term the shift field. Our goal is to obtain the shift field $\widehat{\mathbf{E}}$ in the vicinity of the link associated with the particle move; the boundary of this region is shown as the bold black lines. It is convenient, though not necessary, to take sites $i$ and $j$ to be located in the center of this area (as shown in Fig. 3). We use two parameters $w_{x}$ and $w_{y}$ to specify the shift field area. (In three-dimensional space, one more parameter $w_{z}$ will be needed.) To calculate the shift field, as shown in Fig. 3, we introduce a ghost particle with charge, $q_{i}$ on site $i$, and another particle with charge $q_{i}$ on site $j$. When superposed with the old particle configuration, this pair of charged particles generates the new particle configuration. Then the shift field $\widehat{\mathbf{E}}$ can be obtained by solving the following equations:

$$
\nabla \cdot \widehat{\mathbf{E}}-\rho / \epsilon=0 \text { and } \widehat{\mathbf{E}}=-\operatorname{grad} \widehat{\varphi}
$$

with $\widehat{\mathbf{E}} \cdot \mathbf{n}=0$ on the boundary of the shift field area (the bold lines in Fig. 3), where $\mathbf{n}$ is the normal vector of the

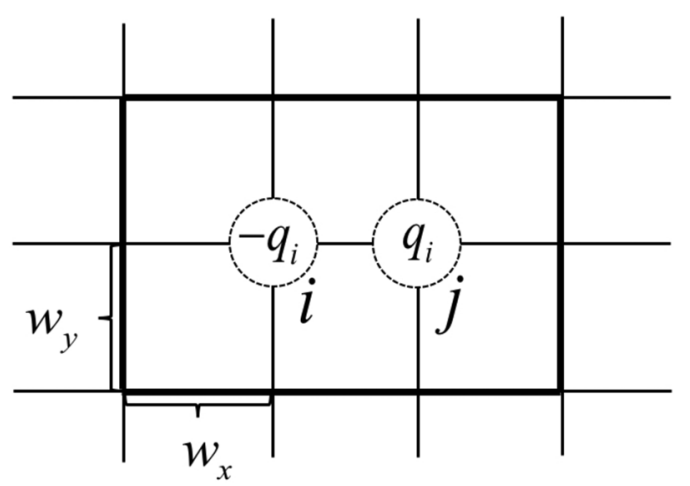

FIG. 3. Schematic for the shift field $\widehat{\mathbf{E}}$ with $w_{x}=w_{y}=1$. Two ghost charged particles are located on sites $i$ and $j$. The bold lines are the boundary of this area.

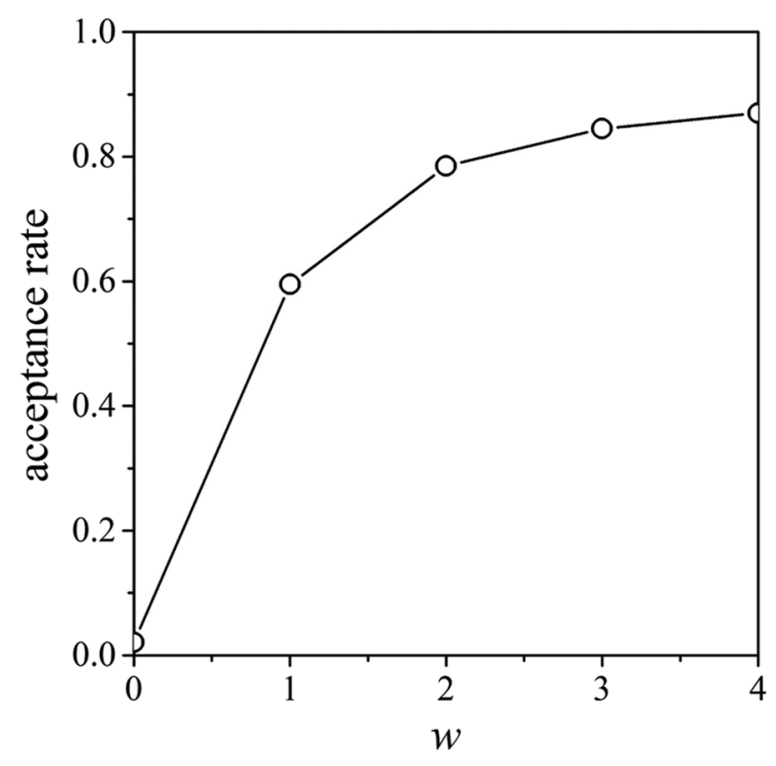

FIG. 4. Acceptance rate for particle moves versus the size parameter $w=w_{x}=w_{y}$ for a system of two univalent particles with opposite charges in a $10 \times 10 \times 10$ simulation box at $T=300 \mathrm{~K}$ with dielectric constant $\epsilon_{\mathrm{r}}=80$. The lattice bond length is $a=2.8 \AA$.

boundary and $\widehat{\varphi}$ is a scalar function. The boundary condition is chosen such that $\widehat{\mathbf{E}}$ is non-vanishing only within the boundary of the shift field area. Note that for a uniform dielectric background, the solution for $\widehat{\mathbf{E}}$ is translationally invariant. For a non-uniform, $\widehat{\mathbf{E}}$ can be trivially obtained from the analogue of the electric displacement $\widehat{\mathbf{D}}=\epsilon \widehat{\mathbf{E}}$ which is again translationally invariant. Therefore, $\widehat{\mathbf{E}}$ only needs to be solved once in the simulation. With $\widehat{\mathbf{E}}$ known, the new field is then $\mathbf{E}_{\text {new }}=\mathbf{E}_{\text {old }}+\widehat{\mathbf{E}}$.

In Fig. 4, we show the acceptance rate as a function of $w=w_{x}=w_{y}$ for a pair of ions in aqueous solution at room temperature. For $w>4$, the acceptance rate no longer changes much with further increase in $w$. In most cases, $w_{x}=w_{y}=1$ is adequate, with an acceptance rate around 0.6.

\section{B. Field update}

Most computer simulations employ PBCs to minimize finite size effects. As pointed out in Ref. 17, and further discussed in Ref. 18, the plaquette updates do not fulfill ergodicity requirements for the field configurations in systems with PBCs. This problem is related with the fact that $\phi=-\overline{\mathbf{E}} \cdot \mathbf{r}$ is a solution of the Poisson equation, where $\overline{\mathbf{E}}$ is an arbitrary constant vector [see Fig. 5(a)], but such a solution cannot be obtained from the plaquette updating scheme. Indeed, it can be shown that any closed-circuit updating scheme, including the worm updates, suffers from this drawback for systems with PBCs. The general reason is that PBC applied in any of the spatial directions generates field configurations that are open in that direction, which cannot be generated by combination of any closed-circuit update; see Fig. 5(b). As a remedy, Maggs and Rossetto proposed adding a uniform background field $\overline{\mathbf{E}}$ to supplement the plaquette updates. ${ }^{18}$

Here we demonstrate that adding a uniform background field does not guarantee ergodicity. This can be understood easily by inspection of the field configuration shown in Fig. 5(b), 


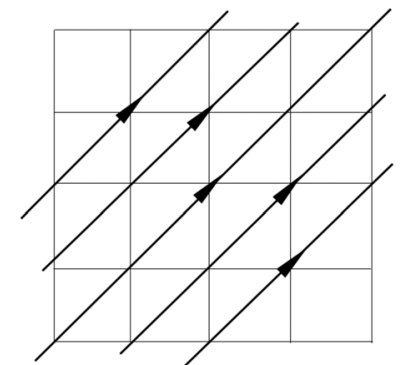

(a)

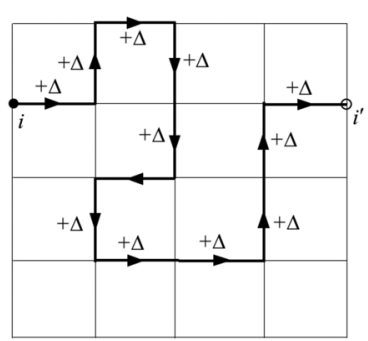

(b)
FIG. 5. Illustration of the (a) uniform background field update and (b) opencircuit field update. In (a), a uniform $\overline{\mathbf{E}}$ is chosen randomly in both magnitude and direction. In (b), a boundary site $i$ is chosen randomly which is connected by a self-avoiding walk path to its periodic image $i^{\prime}$. The field on all the links is modified by adding a random value $\Delta$ chosen within $\left[-\Delta_{0}, \Delta_{0}\right]$.

which clearly cannot be generated by a uniform background field. We note, however, that the supposition of configurations in Fig. 5(b) can generate a uniform background field. Therefore, we propose to supplement the closed-circuit updates with the open-circuit moves such as shown in Fig. 5(b). We note that both the closed-circuit updates and the open-circuit updates shown in Fig. 5(b) meet the constraint $\nabla \cdot \mathbf{E}_{t}=0$. The difference is simply that in the former, the circuits start from site $i$ and end at the same site, whereas in the latter, the circuits start from site $i$ but end at its periodic image position $i^{\prime}$ due to the PBC. The general open circuit takes the form of a random walk starting at $i$ and terminating at $i^{\prime}$. Because of the cancelation due to back-folding, the final path is that of a self-avoiding walk as shown in Fig. 5. It can be shown that such an update scheme satisfies detailed balance.

As an example to demonstrate the lack of ergodicity in the Maggs-Rossetto algorithm and the efficacy of our proposed method, we calculate the probability of ion pair formation for two monovalent ions with opposite sign in a $10 \times 10 \times 10$ simulation box with PBCs. Figure 6 compares results obtained from using the closed circuit worm updates combined with background field updates (blue curve) and those from our new algorithm of using the closed circuit worm updates combined with the open-circuit self-avoiding path updates (red curve). Since the plaquette update is equivalent to the close-worm update, we do not include the result obtained from field updating schemes involving the plaquettes. We also mention that the worm update is more efficient than the plaquette update, consistent with the observation in Ref. 25. The exact result, shown as the black line, is obtained from solving the Poisson equation for each particle configuration and exhaustively enumerates all possible particle configurations with the proper Boltzmann weight. The ion pair probability is calculated as

$$
p_{\text {pair }}=\frac{\sum_{\text {neighbor }} \exp \left[-\beta \mathcal{U}\left\{\mathbf{r}_{+} ; \mathbf{r}_{-}\right\}\right]}{\sum_{\text {all configurations }} \exp \left[-\beta \mathcal{U}\left\{\mathbf{r}_{+} ; \mathbf{r}_{-}\right\}\right]},
$$

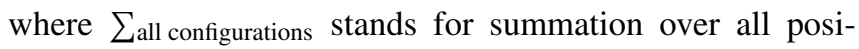
tions of the cation and the anion, $\mathbf{r}_{+}$and $\mathbf{r}_{-}$, respectively, and $\sum$ neighbor restricts summation over configurations in which $\mathbf{r}_{+}$ and $\mathbf{r}_{-}$are nearest neighbors. The energy $\mathcal{U}\left\{\mathbf{r}_{+} ; \mathbf{r}_{-}\right\}$in Eq. (3.4)

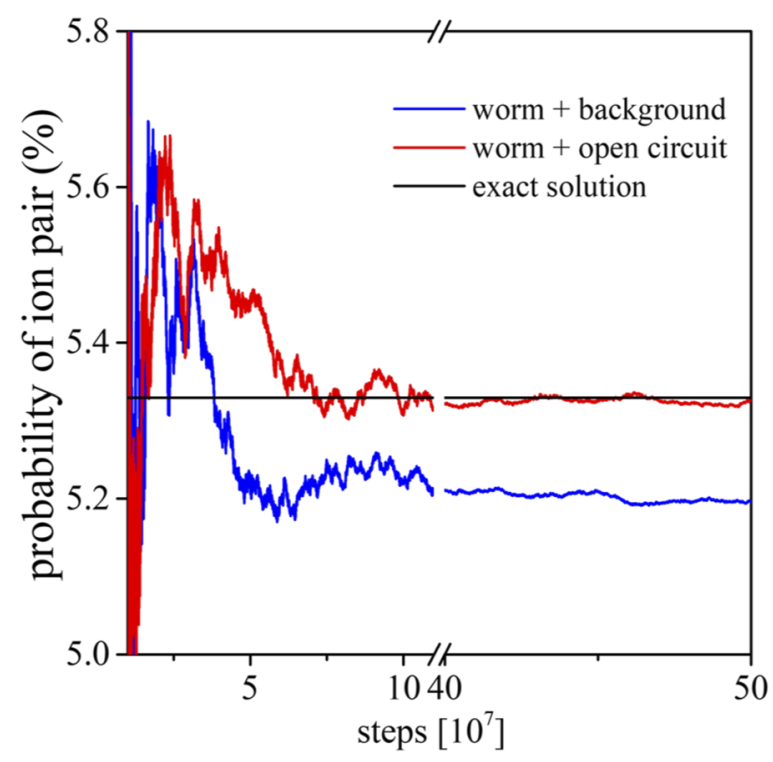

FIG. 6. The probability of ion pair formation for a system of two univalent particles with opposite charges in a $10 \times 10 \times 10$ simulation box at $T=300 \mathrm{~K}$ with dielectric constant $\epsilon_{\mathrm{r}}=80$. The lattice bond length is $a=2.8 \AA$. Blue line: closed-circuit worm updates combined with background field updates; red line: closed-circuit worm updates combined with open-circuit updates; black line: exact solution.

is calculated from

$$
\mathcal{U}\left\{\mathbf{r}_{+} ; \mathbf{r}_{-}\right\}=\frac{\epsilon}{2} \sum_{i<j} E_{i, j}^{2}\left\{\mathbf{r}_{+} ; \mathbf{r}_{-}\right\}
$$

where $E_{i, j}\left\{\mathbf{r}_{+} ; \mathbf{r}_{-}\right\}=\left(\varphi_{i}\left\{\mathbf{r}_{+} ; \mathbf{r}_{-}\right\}-\varphi_{j}\left\{\mathbf{r}_{+} ; \mathbf{r}_{-}\right\}\right) / a . \varphi_{i}\left\{\mathbf{r}_{+} ; \mathbf{r}_{-}\right\}$ can be obtained by solving Eq. (3.2) for any given positions $\left\{\mathbf{r}_{+} ; \mathbf{r}_{-}\right\}$of the ion pair with PBCs.

It is clear from Fig. 6 that there is a finite difference between the results shown in the blue line and the exact result shown in the black line and that this difference does not diminish with increasing the simulation time. By contrast, our new algorithm, with the uniform background field, which is replaced with the open circuits converges to the exact result for sufficiently large number of Monte Carlo steps.

\section{APPLICATION}

As an application of our improved algorithm including both particle moves and field updates, we consider the image charge effects on the ion distribution for electrolytes confined between two dielectric plates with different dielectric constants than the solution. As a result of the dielectric discontinuity, each charge near a surface generates an image charge, which in turn generates an image charge on the apposing plate, and this is repeated ad infinitum. Currently there are no good methods for including these infinite image charges when the separation $D$ is comparable to the Debye screening length $\lambda$. The local algorithm is well suited to treat such systems without any approximation or special difficulty.

We consider two weakly charged plates separated by a distance $D=10 \mathrm{~nm}$, each carrying a surface charge density $\sigma$ $=-0.02 e_{0} / \mathrm{nm}^{2}$, where $e_{0}$ is the elementary charge. The dielectric constants of the plates and solvent are set to be $\epsilon_{p}=2.5$ and $\epsilon_{s}=80$, respectively. For this system, the image charge interaction is repulsive and this image charge repulsion creates a 


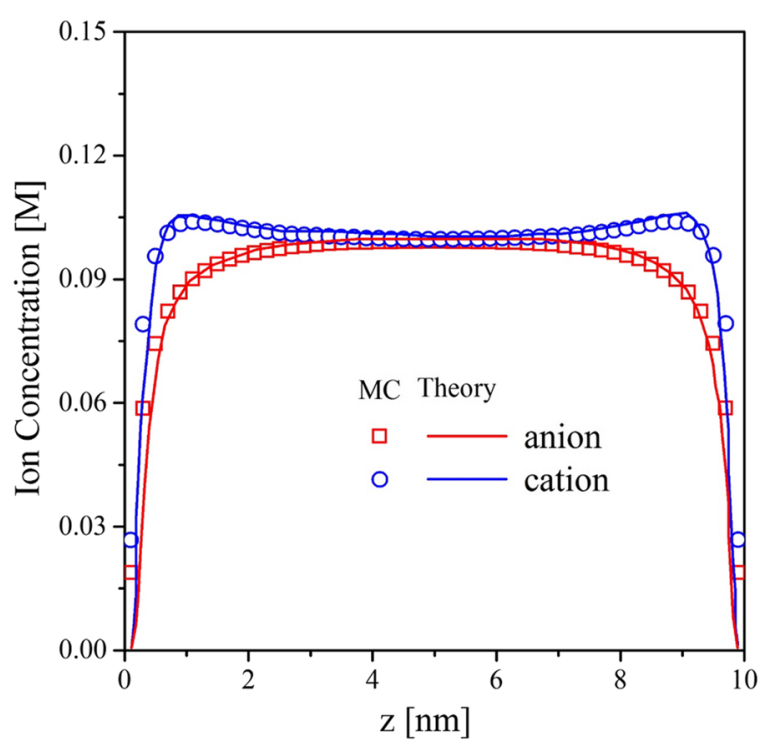

FIG. 7. Ion concentration profile for a 1:1 electrolyte solution between two dielectric plates at $T=300 \mathrm{~K}$. The size of simulation box is $100 \times 100 \times 90$ with length unit $0.2 \mathrm{~nm}$. There are 218 cations and 218 anions to match the total number concentration in Fig. 2(c) of Ref. 26. The symbols are from our simulations and solid lines are from Ref. 26.

depletion layer near the surfaces. This effect is most striking for the counterions (open circles in Fig. 7), since in the absence of image charge repulsions, counterions would accumulate near the charged surface. The depletion for the coions is due to both the surface charge repulsion and the image charge repulsion. The lines in the figure are results obtained by Wang and Wang $^{26}$ using the WKB (Wentzel-Kramers-Brillouin) approximation, ${ }^{27}$ which is valid for low surface charge density and ion concentrations. ${ }^{28}$ The close agreement between the simulation and theoretical results further confirms the validity of the WKB approximation.

\section{CONCLUSIONS}

In this work, we have proposed two improvements on the local Monte Carlo simulation algorithm proposed by Maggs and Rossetto. ${ }^{17}$ By making use of the linear supposition principle for the electric field, we have suggested a new particle move by the construct of a "ghost" charge. This improved particle move significantly enhances the acceptance rate associated with moving a charged particle in the system. We have also demonstrated that the addition of a uniform background field to closed-circuit field update does not guarantee full ergodicity in systems with periodic boundary conditions and suggested instead to supplement the closed-circuit update with the opencircuit update to fully sample the field configurations. As an illustration of the efficacy of our improved algorithm, we have applied our new method to an ionic solution confined between two parallel plates having lower dielectric constant than the intervening solution. The simulation results are in excellent agreement with the previous theoretical work. ${ }^{26}$

In comparison to other existing methods for simulating charge interactions, the Maggs-Rossetto algorithm has a couple of distinct advantages. An obvious advantage is the $O(N)$ computational complexity, which becomes important when the number of charged particles is large. The local nature of the algorithm also makes it suitable for parallelization. More importantly, this local algorithm can treat dielectrically inhomogeneous systems without any approximation or special difficulty. We believe that the improvements proposed in this work will further enhance the efficacy of this local Monte Carlo simulation algorithm and add to its competitiveness in relation to other simulation methods. Finally, our method is quite general and can be used to simulate more challenging systems such as polyelectrolytes near a dielectric interface.

\section{ACKNOWLEDGMENTS}

The Dow Chemical Company is acknowledged for funding and for permission to publish the results. We thank Pengfei Zhang, Anthony Maggs, Issei Nakamura, and Xiaozheng Duan for helpful discussions.

${ }^{1}$ G. Andrés Cisneros, M. Karttunen, P. Ren, and C. Sagui, "Classical electrostatics for biomolecular simulations," Chem. Rev. 114, 779 (2014).

${ }^{2}$ J. V. L. Beckers, C. P. Lowe, and S. W. de Leeuw, Mol. Simul. 20, 369 (1998).

${ }^{3}$ J. W. Perram, H. G. Petersen, and S. W. de Leeuw, Mol. Simul. 65, 875 (1988).

${ }^{4}$ A. Amold and C. Holm, in Advanced Computer Simulation Approaches for Soft Matter II, Advances in Polymer Sciences, edited by C. Holm and K. Kremer (Springer, Berlin, 2005), pp. 59-109.

${ }^{5}$ G. A. Cisneros, V. Babin, and C. Sagui, Biomolecular Simulations (Springer, Berlin, 2013), pp. 243-270.

${ }^{6}$ D. Frenkel and B. Smit, Understanding Molecular Simulation, 2nd ed. (Academic Press, San Diego, 2002).

${ }^{7}$ C. Sagui and T. A. Darden, in Simulation and Theory of Electrostatic Interaction in Solution, edited by L. R. Pratt and G. Hummer (AIP, Melville, New York, 1999), pp. 104-113.

${ }^{8}$ A. Y. Toukmaji and J. A. Board, Jr., Comput. Phys. Commun. 95, 73 (1996).

${ }^{9}$ M. Bolten, H. Dachsel, R. Halver, I. Kabadshow, F. Gähler, F. Heber, J. Iseringhausen, M. Hofmann, M. Pippig, D. Potts, and G. Sutmann, Phys. Rev. E 88, 063308 (2013).

${ }^{10}$ R. W. Hockney and J. W. Easwood, Computer Simulation Using Particles (Taylor \& Francis, Bristol, PA, 1988).

${ }^{11}$ T. Darden, D. York, and L. Pedersen, J. Chem. Phys. 98, 10089 (1993).

${ }^{12}$ U. Essmann, L. Perera, M. L. Berkowitz, T. Darden, H. Lee, and L. G. Pedersen, J. Chem. Phys. 103, 8577 (1995).

${ }^{13}$ J. E. Barnes and P. Hut, Nature 324, 446 (1986).

${ }^{14}$ L. Greengard and V. Rokhlin, J. Comput. Phys. 73, 325 (1987).

${ }^{15}$ K. N. Kudin and G. E. Scuseria, J. Chem. Phys. 121, 2886 (2004).

${ }^{16}$ C. Sagui and T. Darden, J. Chem. Phys. 114, 6578 (2001).

${ }^{17}$ A. C. Maggs and V. Rossetto, Phys. Rev. Lett. 88, 196402 (2002).

${ }^{18}$ A. C. Maggs, J. Chem. Phys. 120, 3108 (2004).

${ }^{19}$ L. Levrel and A. C. Maggs, Phys. Rev. E 72, 016715 (2005).

${ }^{20}$ J. Rottler, J. Chem. Phys. 127, 134104 (2007).

${ }^{21}$ A. Duncan, R. D. Sedgewick, and R. D. Coalson, Phys. Rev. E 71, 046702 (2005).

22 J. Rottler and A. C. Maggs, J. Chem. Phys. 120, 3119 (2004).

${ }^{23}$ K. G. Wilson, Phys. Rev. D 10, 2445 (1974).

${ }^{24}$ F. Alet and E. S. Sørensen, Phys. Rev. E 67, 015701 (2003).

${ }^{25}$ L. Levrel, F. Alet, J. Rottler, and A. C. Maggs, Pramana 64, 1001 (2005).

${ }^{26}$ R. Wang and Z.-G. Wang, J. Chem. Phys. 139, 124702 (2013).

${ }^{27}$ F. P. Buff and F. H. Stillinger, J. Chem. Phys. 39, 1911 (1963).

${ }^{28}$ R. Wang and Z.-G. Wang, J. Chem. Phys. 144, 134902 (2016). 